Cahiers de recherches médiévales

\title{
Vers une revalorisation d'Énée en France
}

Le Séjour d'Honneur d'Octovien de Saint-Gelais

\section{Virginie Dang}

\section{CpenEdition}

\section{Journals}

Édition électronique

URL : https://journals.openedition.org/crm/1593

DOI : $10.4000 / \mathrm{crm} .1593$

ISSN : 1955-2424

Éditeur

Honoré Champion

\section{Édition imprimée}

Date de publication : 15 mars 2003

Pagination : 121-129

ISSN : 1272-9752

Référence électronique

Virginie Dang, « Vers une revalorisation d'Énée en France », Cahiers de recherches médiévales [En ligne] 10 | 2003, mis en ligne le 08 octobre 2007, consulté le 15 décembre 2022. URL : http:// journals.openedition.org/crm/1593; DOI : https://doi.org/10.4000/crm.1593

Ce document a été généré automatiquement le 15 décembre 2022.

Tous droits réservés 


\title{
Vers une revalorisation d'Énée en France
}

\author{
Le Séjour d'Honneur d'Octovien de Saint-Gelais
}

\section{Virginie Dang}

Or est drois que je vous devise

Dou preu Enee et de sa gent

Qui par mer s'en vont nagent

1 Ce passage pourrait provenir du Séjour d'Honneur ${ }^{1}$, poème qu'Octovien de Saint-Gelais a rédigé de 1491 à 1494 et qu'il destinait au roi Charles VIII. Dans ce texte, Énée est l'une des figures exemplaires liées à la quête entreprise par l'Acteur et, à chacune de ses apparitions revient le thème de la navigation. Or, les trois vers susmentionnés initient bien le récit du périple énéen, mais dans l'Ovide moralisé $e^{2}$ et le placent tout entier sous le signe de la mer. Dans les gloses de cette œuvre, le prince troyen est une personnification de « sainte Yglise » exposée aux vicissitudes de l'histoire :

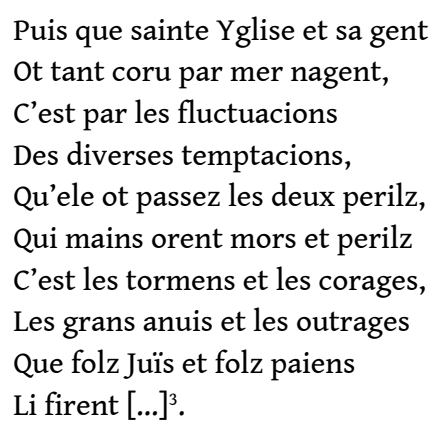

Dans cette perspective, Didon, figure d'opposant, se transforme en "dame Heresie ${ }^{4}$ qui cherche à empêcher Énée-Sainte Église d'atteindre le «port de voire congnoissance $»^{5}$. Le clerc construit la geste d'Énée autour de l'image du navire qui erre sur les flots avant d'arriver à bon port. Cette démarche est emblématique de la méthode employée par l'auteur, ainsi que le constate Renate Blumenfeld-Kosinski : «frequently one dominant image or idea gives shape to an extended allegorization that is generated by semantic fields, such as that of water or navigation. ${ }^{6}$ 
3 Puisque l'Ovide moralisé constitue : «the mythological storehouse for fourteenth- and fifteenth-century poets $»^{7}$, la question se pose de savoir si, comme bien d'autres, Octovien de Saint-Gelais a puisé à cette source pour recréer le héros troyen dans son Séjour d'Honneur. De quelles valeurs Énée est-il porteur, et quel rôle joue l'association privilégiée avec l'élément marin? Pourquoi le couple qu'il forme traditionnellement avec Didon n'y apparaitt-il pas, alors que leur relation fait l'objet de l'une des épîtres appartenant au corpus des Héroïdes ovidiennes, texte qu'Octovien a traduit en 1496 ? Est également évacuée la trahison, vice si typiquement énéen dans la tradition suivant les récits de Dictys ${ }^{8}$ et de Darès ${ }^{9}$. Cette tradition, restée vivace tout au long du Moyen Âge, n'est reprise ni par l'Ovide moralisé ni par Le Séjourd'Honneur. Enfin, à travers le personnage d'Énée, quel type de prince idéal nous est présenté par l'auteur qui réalisera, en 1500, la première traduction de l'Eneide en français?

La métaphore maritime

4 Le quinzième livre de l'Ovide moralisé nous présente l'enseignement de Pythagore. Dans ce passage, la mer, qui, au livre précédent, servait d'élément structurant à l'aventure énéenne, apparaît à nouveau. Sa symbolique renvoie alors à celle de "l'évolution incessante de toutes choses $»^{10}$, qui détermine la vie sur terre. Une telle précision apportée qui plus est dans un traité sur la physique des éléments - nous permet de nuancer le portrait d'Énée ${ }^{11}$. La métaphore maritime ferait du Troyen, non plus tant la personnification de l'Église, de l'ensemble de la communauté chrétienne - comme c'était le cas au livre XIV -, que celle du croyant, de l'individu, soit une figure de la destinée humaine, ayant ses obstacles à surmonter comme autant de tempêtes à traverser.

5 Francine Mora-Lebrun l'écrit déjà, lorsque après avoir rapporté certains commentaires de l'Eneide virgilienne, elle conclut: «Énée n'est plus alors un personnage fictif engagé dans un devenir individuel, mais la figuration de tout esprit humain $»^{12}$. En effet, Mme Mora-Lebrun constate que dans la Consolation de Philosophie de Boèce, la mer se révèle être un «monde mouvant, en proie aux perpétuelles fluctuations que lui imposent la Fortune ${ }^{13}$. Cette image est reprise dans le Roman de la Rose, où Fortune élit domicile sur une île au beau milieu de la mer :
Une roche est en mer seanz
bien parfont, el milieu leanz
qui seur la mer en haut se lance,
contre cui la mer groce et tance
[...] Mais el ne retient nule forme
ainceis se tresmue et reforme ${ }^{14}$

6 Fortune, par le choix de son lieu, prête directement aux flots - qui la contiennent et la portent plus qu'ils ne l'entourent - son caractère changeant et capricieux. Fulgence, dans son Commentaire de l'Eneide, avait appliqué cette métaphore au périple d'Énée. La tempête du livre I y représente la naissance à un monde fait de dangers et d'épreuves ${ }^{15}$, Énée incarnant l'enfant du genre humain. Bernard Silvestre ou de Chartres ${ }^{16}$ inscrit son Commentaire de l'Eneide dans cette lignée. La tempête y symbolise la naissance, avec la chute de l'âme dans le corps. Énée est l'esprit et la mer le corps ${ }^{17}$, corps dont les contraintes épuisent l'esprit, le mettent à mal, tels les flots déchaînés s'acharnant sur le prince et les siens ${ }^{18}$. Le livre II représente l'enfance et le livre III l'adolescence, avec ses hésitations. Le commentateur place la synthèse de sa métaphore, au début du livre $\mathrm{V}$, dans la bouche de cette figure du savoir qu'est la Sibylle : 
PELAGI : mundi vel carnis. Vel pelagus vitam temporalem intelligimus quia eam violentia ventorum exagitat. Ventos autem intelligimus duas temporales fortunas, prosperitatem et adversitatem, que cito veniunt et cito abeuntes transeunt et naves, id est voluntates, ad diversa rapiunt et mentes hominum per hoc mare navigantes in vitiorum naufragium ducunt. ${ }^{19}$

7 Les Troyens n'ont plus à craindre les périls de la mer - qui sont contraintes de la chair faites de pulsions -, son caractère mouvant, instable qui vous ballotte au gré de ses caprices. Les aléas de la Fortune n'ont plus cours au livre VI. C'est de cette façon que le commentaire lit la présence, puis l'absence de la mer, chez Virgile. Dans le Roman d'Eneas, ces années de navigation, entrecoupées par l'escale à Carthage, appartiennent clairement à la phase d'erreur du héros, parce qu'elles précèdent la rencontre avec la Sibylle et Anchise, dont l'enseignement marque un nouveau départ dans la quête d'Eneas ${ }^{20}$.

L'Ovide moralisé insiste sur l'aspect maritime lorsqu'il rapporte l'histoire d'Énée, sans en faire une expression de Fortune à ce moment-là. Le caractère mouvant de la mer est mentionné plus loin dans le texte, l'auteur n'éprouvant pas le besoin de l'intégrer explicitement à sa glose des aventures troyennes, au contraire des commentaires de l'Eneide. La raison pourrait en être que cette métaphore, constituant l'un des topos énéen, ne demandait pas à être longuement développée ; son évocation suffisait pour suggérer l'image d'une Église (représentée par Énée) malmenée par les flots de l'Histoire, telle que la développe la glose.

Énée : figure du traitre ou modèle du prince?

Dans Le Séjour d'Honneur aussi, nous l'avons vu, la navigation est le dénominateur commun à toutes les occurrences d'Énée. Dès le prologue, le prince troyen ouvre la liste des héros, sous l'autorité desquels est placé le périple de l'Acteur. Lorsque l'Acteur, avec l'aide de Bon Advis, se rappelle «les hystoires troyennes » ${ }^{21}$, il cite Énée comme seul protagoniste de cet épisode et ce pour en évoquer les pérégrinations maritimes :

Aussy comment Enee vint a port

Aprés longs jours en la noble Carthage ${ }^{22}$

Bien plus tard, alors que l'Acteur se trouve en compagnie de Sensualité sur la Mer Mondaine, Fol Abus, leur pilote, invoque en pleine tempête, Palinure, aux côtés de Neptune, Triton, Hesperus et Charon. Dans ce lay, dédié aux divinités marines, passeurs et autres guides, le nautonier demande à Palinure, "pere et seigneur du nagent exercice $»^{23}$, de prier Neptune que la mer soit clémente aux voyageurs. Pourtant, Fol Abus sait que le barreur troyen a péri en mer, tué par "Sompnus » ${ }^{24}$, ce « dont Eneas fist maintz doloreux crys $»^{25}$. De plus, Palinure avait guidé les siens en $" s i$ seure police $»^{26}$ jusqu'à Carthage, où Énée se fourvoie, car cette cité n'est pas la nouvelle Troie. Il ne mène pas ses compatriotes jusqu'à cette terre tant attendue qu'est la Lombardie. Fol Abus, lorsqu'il en appelle à lui, ne se contente pas de solliciter la protection d'un nocher expert, mais entend s'identifier à lui, puisqu'il termine en disant : « de qui je vueil imiter la doctrine ${ }^{27}$. Ce processus d'identification n'éclaire-t-il pas, a posteriori, la première évocation d'Énée dans le prologue ? Lorsque l'Acteur tente d'échapper à sa mélancolie, état initial à la quête, il se remémore les " haulx faictz ${ }^{28}$ de certains personnages, dont les premiers cités sont ceux accomplis par Énée ${ }^{29}$. Ne se place-t-il pas, par là, sous l'égide du prince - seul héros de l'Antiquité connoté positivement ${ }^{30}$ - au point de devenir son double? À l'analogie thématique, celle du périple maritime, correspond l'analogie des personnages: l'Acteur suit les traces d'Énée. Ainsi Fol Abus, en invoquant Palinure, fournirait une clé de lecture à la 
navigation sur la Mer Mondaine. L'Acteur ne peut qu'errer en s'y engageant, à l'instar d'Énée, avec Fol Abus à la barre, ce nouveau Palinure. Il ne trouvera pas là sa terre promise.

Plus tard encore, Fol Abus insiste, lorsqu'il tente de " parer d'une ambellie faincte $»^{31}$ la peur de l'Acteur, suscitée par la vision des cadavres flottant sur la Mer, corps inanimés des grands du royaume. Dans cette « ambellie ", il exhorte l'Acteur à faire preuve d'un peu plus de courage :

Il n'est si fort ne si preux conquerant

Qui n'ayt empris cestuy mondain voyage ${ }^{32}$

Et le premier " preux conquérant » cité est à nouveau Énée, qui se trouve confirmé, par là, dans son rôle de principal, voire de seul modèle auprès de l'Acteur. Suivent Brutus, Ulysse, Scipion et César, hautes figures de l'Antiquité gréco-romaine, si célèbres tout au long du Moyen Âge. Aucun acteur, ni de l'histoire récente ni du présent, ne peut être donné en exemple au jeune voyageur, puisque leur état cadavérique témoigne de leur échec. Le passé glorieux devenu mythique, au sens barthien du terme ${ }^{33}$, est utilisé ici pour répondre à la réalité historique, présentée à l'Acteur sous un éclairage des plus sinistres. La dimension mythique qualifie bien Énée, dont Fol Abus rappelle qu'il "bastit [...] une seconde Troye $»^{34}$, cité à l'origine de "Romme la grant " $^{35}$. Et le séducteur à beau jeu de conclure, pour calmer les appréhensions de l'Acteur :

Doncques ne fist Enee fol voyage,

Quant de luy vint ung si noble lignage. ${ }^{36}$

Présenter Énée en ces termes c'est faire de lui le héros fondateur, le prototype même de tout fondateur, qui engendre une nouvelle lignée, plus prestigieuse encore que celle dont il est issu. Par son destin, il devient une figure du prince, mais aussi l'exemple auquel l'Acteur, en quête de lui même, tente de s'identifier. Le cadavre d'Énée ne flotte pas à la surface de la Mer Mondaine. Le prince troyen, ainsi que les autres personnages mythiques, ont réussi à traverser cette mer... sans rester prisonniers dans l'île de Vaine Esperance, où cette dernière mène sa danse. Pourtant, Énée, César et Scipion sont associés, tant au lieu qu'à son activité. Plus précisément, ils ont fait entrer, dans les rangs des danseurs, les protagonistes dont ils ont triomphé, César y a amené Pompée, Scipion Hannibal et Énée Didon.

Relevons qu'Énée ne forme pas un couple avec Didon, au contraire des autres célèbres amants mythologiques, repris comme tels par la littérature médiévale ${ }^{y}$ et que Le Séjour $d^{\prime}$ Honneur cite aussi ensemble: Pâris danse avec "s'amye Helaine " ${ }^{38}$ et Jason avec Médée ${ }^{39}$. Didon participe au mouvement, mais seule, "tout en courroux nee», "se complaingnant du faulx parjure Enee/Et de sa nef $[. ..] »^{40}$.

Octovien ne voit pas en Énée un amant. Il ne fait pas d'Énée et Didon un couple. La réflexion menée sur le prince ne prend pas en considération son aventure à Carthage. Il n'y a pas d'enseignement à tirer de cette histoire sentimentale - du moins pas concernant Énée -, au contraire de celles vécues par les autres couples mythologiques, qui eux tournoient dans cette danse désespérée. Didon virevolte à leurs côtés, car elle participe à leur désespoir par son "courroux", à travers lequel s'exprime la subjectivité d'une femme blessée. Octovien anticipe ici le ton qui caractérisera le discours de Didon dans sa traduction des Héroïdes d'Ovide. Mais dans Le Séjour d'Honneur, le narrateur n'adhère pas au jugement de Didon sur Énée ; il se contente de rapporter ce qu'il voit et entend, laissant la reine seule, livrée à elle-même, donc ne pouvant s'en prendre qu'à elle pour son triste sort. 

puisque cette reine est une perdante, alors que le prince est un vainqueur. En effet, dans Le Séjour d'Honneur, Octovien, lorsqu'il s'attarde sur le personnage d'Énée, met l'accent sur la figure de la destinée humaine qui, certes, vogue sur les flots, mais finit par débarquer sur le sol de la nouvelle Troie et voit donc que la Fortune joue en sa faveur.

17 À sa descente de la nef de Fol Abus, l'Acteur parvient à la Forest des Advantures. C'est dans ce lieu que se trouve confirmé le caractère de vainqueur attribué à Énée. Au moment d'y pénétrer, l'Acteur demande à Sensualité de lui révéler le mystère sur la fontaine de larmes située à l'orée de cette forêt. Le guide raconte alors comment Adam et Ève furent les premiers à être « gettéz a sort et advanture [...] a peines et travaulx [...] a million de maulx $»^{41}$. Cain et Abel leur succèdent, puis les cités de Babylone, de Sodome et Gomorrhe, les victimes du Déluge et les ennemis vaincus par Abraham ; «le vouloir du tressouverain Juge $»^{42}$ châtie, punit. Cette même loi, dont la logique échappe à l'entendement humain, s'applique aussi à la geste énéenne, qui constitue, une fois encore, un des épisodes de l'histoire de l'homme.

Le pouvoir de Priam " tourna en advanture $»^{43}$, tant et si bien qu'il « vit ses filz mors et sa cité par terre $\aleph^{44}$. Quel péché a commis ce souverain? Quel fut celui de Turnus, qui "perdit son butin par les Troyens et fatalle ordonnance ${ }^{45}$ - dont les arcanes restent insondables - alors qu'il est qualifié d'« homme de fier couraige $»^{46}$ ? Ce destin est toujours favorable à Énée :

En ce quartier Enee ediffia

Aprés sa fuyte et son long navigage

La neufve Troie et en fin deffia

Le duc Turnus ${ }^{47}$

Dans Le Séjour d'Honneur, Énée traverse, avec un invariable bonheur, les flots de la mer, de sorte que sa navigation semble se réduire à un prélude qui mène à l'acquisition de la terre promise, où il pose les bases de la nouvelle Troie. Il a surmonté, sans crainte et avec succès, toutes les épreuves. Cette mer n'en perd-elle pas ainsi tout caractère fluctuant, imprévisible, devenant par là une manifestation, non tant de la Fortune que du Destin, voire de la Providence ? Énée n'est pas ici n'importe quelle figure de la destinée humaine, mais bien l'élu, du moins selon Abus et Sensualité. Nous retrouvons là le motif de base du texte virgilien ${ }^{48}$, alors que la difficile acquisition des vertus royales constituait le thème central du Roman d'Eneas ${ }^{49}$.

Mais bâtir une cité, fonder un lignage sont-ce là les enjeux de la quête que poursuit l'Acteur du Séjour? Le modèle qu'y offre Énée n'est-il pas par trop politique, véhiculant des valeurs difficilement applicables à un individu qui se cherche? La victoire de ce prince est rattachée aux notions de gloire et de puissance terrestres, alors que L'Acteur recherche une réalisation personnelle, dont il comprendra qu'elle ne peut être que d'ordre spirituel. Dans cette optique, seul importe l'Énée de l'errance. Ce modèle disparaît dès que l'Acteur se met en chemin pour trouver la Cour d'Honneur. Énée ne joue un rôle que dans la première partie du récit, lorsqu'il est évoqué dans les discours tenus par ces deux allégories négatives, ces deux figures de la séduction trompeuse que sont Sensualité et Fol Abus. Énée correspond donc à la phase d'erreur dans le parcours effectué par l'Acteur. Il fait bien figure d'exemple, mais cet exemple est biaisé. La quête de l'Acteur est autre. 
Bien qu'il ne puisse pas servir de référence pour l'Acteur, Énée bénéficie d'une image positive dans Le Séjour d'Honneur. Le regard que porte Octovien de Saint-Gelais sur le héros troyen se révèle très personnel, en ce qu'il se démarque clairement de celui de ses contemporains. En effet, pour une grande majorité des auteurs du XVe siècle, Énée fait office de triste sire, accusé de trahison tant envers les siens que vis-à-vis de Didon. Les textes historiques adressés au prince et, par là, susceptibles de fournir un modèle royal, présentent Énée, avec insistance, sous les traits peu flatteurs du traitre, en particulier à sa patrie.

Jean de Courcy, chevalier qui prit la plume seulement sur ses vieux jours, s'attarde, dans sa compilation d'histoire ancienne intitulée $L a$ Bouquechardière ${ }^{50}$, longuement sur les aventures du prince troyen, qui commencent avec la chute de la cité de Priam. L'auteur place dans la bouche d'Hécube des propos accusateurs envers le fils d'Anchise. Troye en cendres, la reine « regarde » Énée qui conduit les Grecs :

Si lui reprocha lors la grande trahison qui contre son seigneur avoit pourchassee et contre tout le peuple de la cite de Troye. De faulcete adonc l'appella ${ }^{51}$

Dans le mystère, intitulé Istoire de la Destruction de Troye la Grant ${ }^{52}$, qu'il dédie à Charles VII, Jacques Milet prête un discours diffamatoire envers ce prince, non seulement à Hécube ${ }^{53}$ mais également à son époux, Priam ${ }^{54}$, ainsi qu'à Énée, qui se mortifie et jure de ne plus recommencer :

Cest raison que pour mes meffaiz

Jendure le pugnissement

Las ie faiz aux dieux bon serment

Que iamais en nulle facon

Je ne comettray trahison ${ }^{55}$

Gauvain Quiéret, petit noble picard, dans son Trialogue $e^{56}$, dénonce également auprès de son souverain, Louis XI, le vice qui provoque la chute des grands empires. En effet, ce n'est pas par les armes que Troie fut prise mais grâce à :

la traÿson des Troyens, qui trayrent leur roy lige et le livrairent, luy et sa noble cité et ceulx meismes de son propre sang, et ou il se fioit le plus comme, Anchisés et Anthenor, Eneas et leurs consors. ${ }^{57}$

Il n'y a pas que les clercs et les chevaliers pour dénoncer le prince troyen. Le mauvais renom de ce dernier se retrouve sous la plume d'un marchand, Jean Panier. Dans son Epistre des Ronmains ${ }^{58}$, celui-ci fait remonter la corruption des Romains, leur avidité, à Énée. C'est par lui, « le traictre $»^{59}$, que «Prïan vit sa cité pillee $»^{60}$. Suite à quoi, il fonda Rome et «l'enrichit du pillage de Troye ${ }^{61}$.

Si la trahison d'Énée est évoquée dans Le Séjour d'Honneur, c'est dans les propos de Didon, dictés par sa subjectivité d'amante désespérée. Le jugement que le narrateur porte sur Énée n'est pas entaché par ces considérations. Ce vice ne saurait qualifier le prince. Et même si Fol Abus et Sensualité ne sont pas des guides très recommandables, Octovien de Saint-Gelais, ouvre, par la louange d'Énée, la voie à une revalorisation du héros troyen. Il est déjà l'élu; le voyage en mer, sans constituer un réel obstacle, sert d'épreuve qui le confirme dans son statut.

Une telle réhabilitation devait permettre, dans un deuxième temps, de présenter le prince troyen comme modèle au souverain, pour les guerres d'Italie, modèle et aïeul sur les traces duquel marcher pour aller récupérer les terres ancestrales. L'image du fondateur, si elle n'est pas encore valable pour le clerc-Acteur du Séjour d'Honneur, l'est bel et bien pour le monarque qui entreprend ces guerres. 


\section{NOTES}

1.Éd. F. Duval, Genève, Droz, 2002, p. 534.

2.Éd. C. de Boer, Amsterdam, Noord-Hollandsche Uitgevers-Maatschappij, 1938, t. 5, Livre XIV, p. 18, vv. 302-304.

3.Idem, p. 24, vv. 527-536.

4.Idem, p. 25, v. 571.

5.Idem, v. 541.

6.Reading Myth: Classical Mythology and its Interpretations in Medieval Literature, Stanford, University Press, 1997, p. 119.

7.Idem, p. 215. Voir aussi le récent Cahiers de Recherches Médiévales : « Lectures et usages d'Ovide (XIII'-XV' siècles) », 9, 2002.

8.Ephemeridos Belli Troiani libri, éd. W. Heisenhut, Leipzig, Teubner, 1958.

9.De excidio Troiae historia, éd. F. Meister, Leipzig, Teubner, 1873.

10.Citation tirée de A. Pairet, Les mutacions des fables. figures de la métamorphose dans la littérature française du Moyen Âge, Paris, Champion, 2002, p. 130.

11.Démarche que l'Ovide moralisé n'a pas explicitement entreprise.

12. L'« Eneide " médiévale et la naissance du roman, Paris, P. U. F., 1994, deuxième partie : "L'« Eneide » des Chartrains », chapitre VIII : « La médiation poétique des Noces de Martianus Capella et de la Consolation de Boèce », p. 160.

13. Idem, p. 161.

14. Éd. F. Lecoy, Paris, Champion, 1976 (1965), t. I, p. 181, vv. 5891-5894 et vv. 5903-5904.

15. Fulgenzio, Commento all'Eneide, a cura di Fabio Rosa, Milano, Luni Editrice, 1997, pp. 58-59.

16. Pour la question de l'attribution voir F. Mora-Lebrun, L'« Eneide » médiévale..., deuxième partie, pp. 93-97.

17. mare corpus humanum in J. W. Jones and E. F. Jones, The Commentary on the first six Books of the Aeneid of Vergil commonly attributed to Bernardus Silvestris, Lincoln and London, University of Nebraska Press, 1977, Livre 1, p.10, 1.15.

18.Idem, Livre 1, p.10 et sq.

19.Idem, Livre V, pp. 49-50 : « La mer : le monde ou la chair. Par la mer nous entendons la vie temporelle, parce que cette dernière est agitée par la violence des vents. Par les vents nous entendons les deux fortunes temporelles, la prospérité et l'adversité, qui viennent rapIdement, s'en vont aussi vite, entraînent les navires, c'est-à-dire les volontés, de divers côtés et mènent les esprits des hommes qui naviguent sur cette mer au naufrage des vices ». Cette traduction est tiré de F. Mora-Lebrun, $L$ ' Eneide» médiévale..., op. cit., p. 161

20.Pour cette question je me permets de renvoyer à mon article : « De la lâcheté du guerrier à la maîtrise du prince : Enée à la conquête du pouvoir », Le Moyen Âge, CVII, 2001, pp. 9-28.

21.Éd. citée, Livre I, p. 118, v. 110. 
22.Idem, vv. 115-116.

23.Livre II, iii, p. 172, v. 37.

24.Idem, v. 46.

25.Idem, v. 48.

26.Idem, v. 40.

27.Idem, v. 50.

28.Livre I, i, p. 118, v. 98.

29.Idem, v. 115.

30.Un seul autre nom apparaît, mais connoté négativement : «l'outrage Pompeÿan » Idem, v. 122-123.

31.Livre II, ix, p. 186, v. 34 .

32.Livre II, x, p. 189, vv. 70-71.

33.R. Barthes, Mythologies, Paris, Seuil, 1957, « Le mythe, aujourd'hui », pp. 191-247.

34.Livre II, x, p. 187, vv. 23-24.

35.Idem, p. 188 , v. 30.

36.Idem, vv. 31-32.

37.Voir à ce sujet D. Poirion, Le Poète et le prince. L'évolution du lyrisme courtois de Guillaume de Machaut à Charles d'Orléans, Genève, Slatkine Reprints, 1978 (Grenoble, 1965), chap. XIV : « La lucidité du sage : raison et résignation », p. 588 ; ainsi que mon article : «Enée et Didon chez Eustache Deschamps : de la mythologie à la moralisation politique » (à paraître).

38.Livre II, xxxi, p. 217, v. 44.

39.Idem, vv. 49-56.

40.Livre II, xxxi, p. 218, vv. 83-85.

41.Livre III, ix, p. 295, vv. 12-14.

42.Idem, p. 297, v. 78.

43.Idem, p. 299, v. 147.

44.Idem, v. 151.

45.Idem, p. 300, vv. 167-168.

46.Idem, v. 164.

47.Idem, vv. 161-164.

48.Eneide, éd. J. Perret, Paris, Les Belles Lettres, 1995 (1977), Livre I, p. 5, v. 1-3.

49.Voir mon article : « De la lâcheté du guerrier à la maîtrise du prince... » cité à la note 20.

50.Paris, B. N., ff. 329 et Genève, B. P. U., ms. fr. 70/1 et 2. Voir à propos de ce texte l'article de B. Chancel, « Les manuscrits de la Bouquechardière de Jean de Courcy », Revue d'histoire des textes, 17, 1987, pp. 219-290.

51.Genève, B. P. U., ms. fr. 70/1, Livre II, chap. XXXVI, folio 147v.

52.Repr. de l'incunable de 1484, E. Stengel, Leipzig-Marburg, 1883.

53.Éd. cit., pp. 417-418, vv. 26819-26869.

54.Éd. cit., p. 416, vv. 26751-26766, et plus particulièrement : v. 26760.

55.Éd. cit. p. 433, vv. 27910-27914.

56.Paris, Bibliothèque de l'Arsenal ms 4655. Cf. M. -R. Jung, « Le Trialogue de Gauvain Quiéret (MS. Arsenal 4655) », Studi Provenzali e Francesi 86/87 (= Romanica Vulgaria Quaderni 10/11) 1990, pp. 209-231.

57.Manuscrit cité, folio $152^{\mathrm{r}}$.

58.Éd. A. Menichetti, « L'inedita « Epistre des Ronmains » del cod. vat. Reg. lat. 1323 », Cultura neolatina, 26, 1966, p. 158-198. 
59.Éd. cit., p. 178, v. 33.

60.Idem, v. 34

61.Idem, p. 179, v. 42.

62.Paris, B. N. ff. 861

\section{AUTEUR}

VIRGINIE DANG

Université de Lausanne 